\title{
TIME OF ARRIVAL 3-D POSITION ESTIMATION USING MINIMUM ADS-B RECEIVER CONFIGURATION
}

\author{
A. S. Yaro ${ }^{1,}{ }^{*}$, A. Abdulaziz ${ }^{2}$, S. Salisu ${ }^{3}$, S. Muhammad ${ }^{4}$ and S. A. Eleruja ${ }^{5}$ \\ 1,4,5, DePt. OF Electrical AND COMPUTER ENGR., AHMAdu BEllo University, ZARIA, KADUNA STATE, NIGERIA

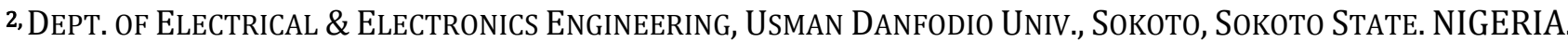 \\ 3, FACULTY OF ElECTRICAL ENGINEERING, UNIVERSITI TEKNOLOGI MALAYSIA, 81310, SKUDIA, JOHOR, MALAYSIA. \\ E-mail addresses:1yaroabdulmalik@yahoo.com, 2 abdul2140@gmail.com,3s.salisu@live.com, \\ 4 coolsaeed201@yahoo.com, 5 doguwanakhadija@gmail.com
}

\begin{abstract}
The location from which a signal is transmitted can be estimated using the time it takes to be detected at a receiver. The difference between transmission time and the detection time is known as time of arrival (TOA). In this work, an algorithm for 3-dimensional (3-D) position estimation (PE) of an emitter using the minimum receiving stations configuration was developed. The TOA measurements estimated from three automatic surveillance dependent broadcast (ADS-B) receivers are used to evaluate the performance of the PE algorithm. The result shows that the triangular ADS-B receiver configuration is capable of 3-D PE for emitter locations within $190 \mathrm{~km}$ coverage radius and that the altitude estimation error is $2 \mathrm{~km}$ higher than the horizontal coordinate estimation error. Within the 3-D PE coverage of the ADS-B receiver, the maximum horizontal and altitude error expected are $0.12 \mathrm{~km}$ and $2.2 \mathrm{~km}$ respectively.
\end{abstract}

Keywords: TOA, ADS-B, position estimation, minimum configuration, surveillance

\section{INTRODUCTION}

Passive time of arrival (TOA) based position estimation (PE) system for air traffic surveillance purposes, estimate the emitter location in two stages [1]. The first stage involves estimating the TOA of the signal at each receiver. The TOA is the time taken by an electronic magnetic emission to propagate from the transmitter to the receiver. There are several techniques reported for estimating TOA but the classical techniques used in the air traffic surveillance system is the leading-edge detection approach using threshold crossing $[3,4]$. This involves getting the time instance at which first leading pulse of the signal overcomes the assigned threshold. The time is obtained from a clock the receiver is equipped with. Another approach is the maximum likelihood estimator (MLE) method [5]. The use of the MLE method with time-stamp to estimating TOA of ADS-B packets in a semi-passive scenario was presented in [5]. The received ADS-B packet is correlated with the know preamble structure and the time at which the maximum peak occurs is recorded. This recorded time corresponds to the TOA of the received ADS-B packet. Other techniques for TOA estimation include shift invariant technique [6] and super resolution technique $[7,8,9]$ which are based on frequency domain deconvolution.

The second stage involves using the estimated TOA measurements from the first stage as inputs to a PE algorithm to estimate the emitter position. $N$ number of ADS-B receivers will result to $N$ number of TOA measurement Depending on the receivers used, 2D or 3D PE of the emitter is possible. For a 3D PE, a minimum of three receivers are required [1]. $N$ number of TOA measurement will result in $N$ number of circle equations with each circle equation representing a nonlinear equation between the TOA measurements and emitter position. To solve for the emitter position, there is need to have a linear relationship between the two parameters (TOA measurements and emitter position). Several articles have reported on how the linear relationship between the two parameters is obtained which resulted in difference PE algorithm grouped into: linear PE and nonlinear PE algorithm [1]. The TOA based non-linear PE algorithm involves the use of linearization techniques to obtain the linear relationship between

\footnotetext{
* Corresponding author tel: +234-806-925-4680
} 
the two parameters and a random initial emitter position is inputted which is then refined using iteration methods such as Gauss-Newton method to obtain the final emitter position $[1,10]$. This approach suffers from convergence if the initial emitter position is far from the actual emitter position. The TOA based linear PE algorithm involves algebraic manipulation of the $N$ number circle equations to obtain a linear relationship between the two parameters [1, 11, 12]. It has no convergence issue as it does not require initial emitter position to be inputted and refined. In this work, the linear PE algorithm is adopted for the emitter PE process. Other forms of PE algorithm used in emitter PE are multilateration [13] and multiangulation [14] which are based on estimating time difference of arrival (TDOA) of the signal at receiving station pairs and angle of arrival (AOA) respectively.

In our earlier work [15], an ADS-B receiver was designed and its performance in decoding ADS-B packet was determined in term of bit error rate (BER) and packet error rate (PER). The ADS-B receiver proposed in [15] is used in obtaining TOA measurements based on time-stamp. The TOA measurements are then used in evaluating the performance of a developed 3-D TOA based PE algorithm using the minimum number of ADS-B receivers. The PER performance of the ADS- $B$ receiver is used in determining the 3-D PE coverage of the TOA PE system. Simulation results for analysis are obtained using Monte Carlo with ADS-B receivers in triangular configuration.

The rest of the paper is organized as follows. Obtaining the TOA measurement for PE using the ADS-B receiver is described in section 2 . Section 3 gives a description of the TOA-based PE algorithm. Simulation result and discussion is presented in section 4 which is followed by conclusion in section 5 .

\section{ADS-B TOA MEASUREMENT FOR PE}

When an ADS-B packet is received, the time at which the packet is received is time-stamped on the ADS-B packet. If the transmission time is known and there is perfect time synchronization between the emitter and all the ADS-B ground receiving station (GRS)s, the TOA of the signal is calculated as:

$$
\tau \approx\left|T_{\text {stamp }}-T_{\text {trasmit }}\right|
$$

where $T_{\text {stamp }}$ is the time-stamp on the ADS-B packet and $T_{\text {transmit }}$ is the transmission time on the ADS- $B$ packet. Since the time-stamp approach is used for TOA estimation, error in TOA measurement is related to the receiver time-stamp resolution. Time-stamp resolution varies for different receiver manufacturer and ranges from 2 nsec to 20 nsec $[16,17]$.

After the ADS-B packet is received, a cyclic redundancy check (CRC) is carried out to validate the correctness of received packet $[15,18,19]$. The ability of an ADS-B receiver to carry out such validation process of ADSpacket is measured through its PER which ranges from 0 to 1 . The PER value 0 means there is no error in the ADS-B packet while 1 PER value mean there is error in the ADS-B packet. An ADS-B packet is valid if the receiver decodes the packet with a PER value of 0 . If the PER $=0$, timestamp on an ADS-B packet can be used for TOA estimation and PE. Therefore, for 3-D PE to be performed, all 3 ADS-B receivers must decode the ADS$B$ packet with PER of 0 . The estimated TOA measurements are used to determine the position of the emitter in the next section.

\section{TOA-BASED PE ALGORITHM}

In this section, a detail description of the TOA PE localization algorithm is presented. The TOA measurement from Eq. (1) at each ADS-B receiver is assumed to contain error. Let $\hat{\tau}_{i}$ be the estimated TOA measurement at the $i$-th ADS-B receiver with coordinates $\left(x_{i}, y_{i}, z_{i}\right)$. The estimated TOA at the $i$-th ADS-B receiver is related to the emitter position $\mathbf{x}=$ $(x, y, z)$ as shown in Eq. (2).

$$
\hat{\tau}_{i}=\frac{\sqrt{\left(x-x_{i}\right)^{2}+\left(y-y_{i}\right)^{2}+\left(z-z_{i}\right)^{2}}}{c}
$$

where $c$ is the speed of light in vacuum given as $3 \times 10^{5} \mathrm{~km}$.

From Eq. (2), the estimated TOA ( $\hat{\tau}$ )and the emitter position (x) have a non-linear relationship. By taking the square of both side in Eq. (2), the resulting expression is

$$
\left(\hat{\tau}_{i}-c\right)^{2}=\left(x-x_{i}\right)^{2}+\left(y-y_{i}\right)^{2}+\left(z-z_{i}\right)^{2}
$$

Eq. (3) can be further simplified resulting to

$$
\begin{gathered}
\left(\hat{\tau}_{i}-c\right)^{2}=\left(x^{2}+y^{2}+z^{2}\right)-\left(2 x x_{i}+2 y y_{i}+2 z z_{i}\right) \\
+\left(x_{i}^{2}+y_{i}^{2}+z_{i}^{2}\right)
\end{gathered}
$$

Let

$\hat{R}_{i}=\left(\hat{\tau}_{i} \times c\right)^{2}, . R=x^{2}+y^{2}+z^{2}, K_{i}=x_{i}^{2}+y_{i}^{2}+z_{i}^{2}(5)$ Substituting Eq. (5) into Eq. (4), the resulting expression is

$$
\hat{R}_{i}^{2}=R-2\left(x x_{i}+y y_{i}+z z_{i}\right)+K_{i}
$$

To performance 3-D PE using TOA measurements, a minimum of 3 ADS-B receivers are needed. Therefore, the TOA equations for $1 \leq i \leq 3$ are

$$
\begin{gathered}
\hat{R}_{1}^{2}=R-2\left(x x_{1}+y y_{1}+z z_{1}\right)+K_{i} \\
\hat{R}_{2}^{2}=R-2\left(x x_{2}+y y_{2}+z z_{2}\right)+K_{2} \\
\hat{R}_{3}^{2}=R-2\left(x x_{3}+y y_{3}+z z_{3}\right)+K_{3}
\end{gathered}
$$


Subtracting both Eq. (7b) and Eq. (7c) from Eq. (7a) to eliminate the variable $R$ as it is a function of the emitter position which is unknown for a passive PE system. This results into two equations, according to [12], which are:

$$
\begin{gathered}
\hat{R}_{1}^{2}-\hat{R}_{2}^{2}=2 x X_{21}+2 y Y_{21}+2 z Z_{21}+K_{12} \\
\hat{R}_{1}^{2}-\hat{R}_{3}^{2}=2 x X_{31}+2 y Y_{31}+2 z Z_{31}+K_{13}
\end{gathered}
$$

where

$$
\begin{aligned}
X_{j 1}=x_{j}-x_{1}, Y_{j 1} & =y_{j}-y_{1}, Z_{j 1}=Z_{j}-Z_{1} \text { and } K_{1 j} \\
& =K_{1}-K_{j} \text { for } 2 \leq j \leq 3
\end{aligned}
$$

Using Eq. (8) and Eq. (9), the emitter position $\mathbf{x}=$ $(x, y, z)$ can be obtained given the TOA measurements and ADS-B receiver coordinates. To solve for the emitter position, make $y$ subject of formula in Eq. (8), which results to

$$
y=\frac{0.5\left(R_{1}^{2}-R_{2}^{2}-K_{12}\right)}{Y_{21}}-x \frac{X_{21}}{Y_{21}}-z \frac{Z_{21}}{Y_{21}}
$$

Substitute $y$ in Eq. (10) into Eq. (9) and making $x$ subject of the formula results into

$$
x=Z \times A+B
$$

Where

$$
\begin{gathered}
A=\left(\frac{Z_{21} Y_{31}-Y_{31} Y_{21}}{X_{31} Y_{21}-X_{21} Y_{31}}\right), \\
B=\frac{\left(0.5 Y_{21}\left(R_{1}^{2}-R_{3}^{2}-K_{13}\right)\right)-\left(0.5 Y_{31}\left(R_{1}^{2}-R_{2}^{2}-K_{12}\right)\right)}{X_{31} Y_{21}-X_{21} Y_{31}}
\end{gathered}
$$

Eq. (11) shows the $x$ coordinate of the emitter as a function of the altitude $z$. Substituting $x$ in Eq. (11) into Eq. (10), the resulting expression after simplification is

$$
y=C-z D
$$

Where

$$
C=\frac{0.5\left(R_{1}^{2}-R_{2}^{2}-K_{12}\right)}{Y_{21}}-A \frac{X_{21}}{Y_{21}} \text { and } D=B \frac{X_{21}}{Y_{21}}+\frac{Z_{21}}{Y_{21}}
$$

Eq. (12) shows the zcoordinate of the emitter as a function of the altitude $z$. Substituting $x$ and $y$ in Eq. (11) and Eq. (12) respectively into Eq. (7a), simplification results into a second order quadratic equation as a function of the emitter altitude $z$ as shown in Eq. (13).

$$
Z^{2} P+z Q+R=0
$$

Where

$$
\begin{aligned}
P=D^{2}+B^{2}+1 & , Q \\
& =2 A B-2 D C-2 x_{1} B-2 y_{1} D \\
& +2 z_{1} \text { and } R \\
& =A^{2}+C^{2}-2 x_{1} A-2 C y_{1}+K_{1}-R_{1}^{2}
\end{aligned}
$$

Solving for $z$ in Eq. (13) gives

$$
z=\frac{-Q \pm \sqrt{Q^{2}-4 P R}}{2 P}
$$

Since the altitude of the emitter cannot be a negative value, thus, the altitude of the emitter is

$$
z=\frac{-Q+\sqrt{Q^{2}-4 P R}}{2 P}
$$

Using the solution of $z$ from Eq. (15), substitute into Eq. (11) and Eq. (12) to obtain the corresponding $x=z A+B$ coordinate and $y=C-z D$ coordinate respectively which are:

\section{SIMULATION RESULT AND DISCUSSION}

Position root mean square error (RMSE) is used as the PE performance measure. The horizontal and altitude RMSE respectively are mathematically expressed as

$$
\begin{aligned}
& H_{r m s e}=\sqrt{\frac{\sum_{i-1}^{N}\left(\left(x-\hat{x}_{i}\right)^{2}+\left(y-\hat{y}_{i}\right)^{2}\right)}{N}} \\
& \text { Alt }_{\text {rmse }}=\sqrt{\frac{\sum_{i=1}^{N}\left(z-\hat{z}_{i}\right)^{2}}{N}}
\end{aligned}
$$

where $(x, y, z)$ is the known emitter position and $\left(\hat{x}_{i}, \hat{y}_{i}, \hat{z}_{i}\right)$ is the emitter position at the $i$-th Monte Carlo realization. Monte Carlo simulation results were obtained after 200 realizations.

Surveillance system used in aircraft monitoring displaces the position of emitters in cylindrical coordinate systems, that is, range $(R)$ bearing $(\theta)$ and altitude $(z)$ [20]. Conversion from cylindrical coordinate system to the rectangular coordinate system can be done using Eq. (17).

$$
\begin{gathered}
x=R \times \cos (\theta) \\
y=R \times \sin (\theta)
\end{gathered}
$$

The distribution of the ADS-B receivers for PE analysis are shown in Figure 1.

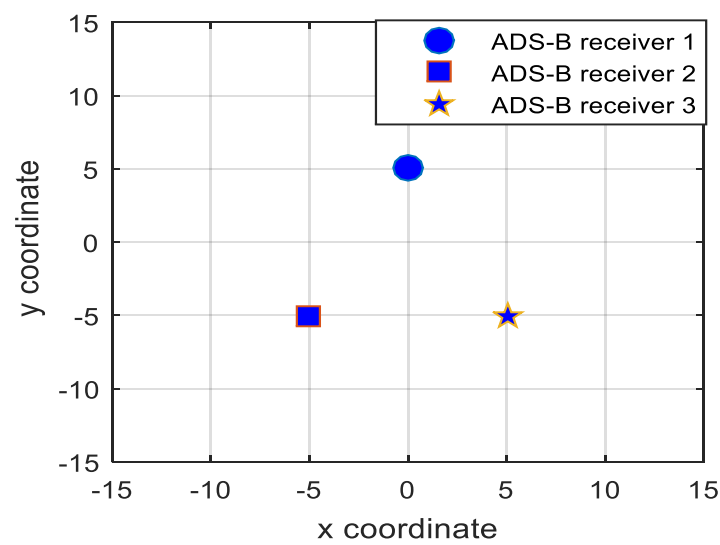

Figure 1: Triangular distribution configuration of ADSB receivers [5 km separation]

Figure 1 shows the triangular distribution configuration of the three ADS-B receivers. Each ADS-B receiver is placed at a vertex of an equilateral triangle with a side length of $5 \mathrm{~km}$. According to Chan et al. [21], simple configurations such as squares and equilateral triangles result in better PE accuracy. Based on what was suggested in [21], the triangular configuration as shown in Figure 1 is adopted and the PE accuracy of the 
TOA-based PE system is determined for this configuration.

\subsection{ADS-B receiver PER Versus Signal to Noise Ratio (SNR) Relationship}

In this paper, the ADS-B receiver proposed in [15] is used for the TOA estimation. The performance of the receiver in terms of PER versus SNR is shown in Figure 2. It is seen that the PER decreases with increase in the SNR from $11 \mathrm{~dB}$ to $20 \mathrm{~dB}$. At SNR of $10 \mathrm{~dB}$, the PER is 1 meaning that the ADS-B packet received at that SNR value contains an error. At SNR above $20 \mathrm{~dB}$, the PER is equal to 0 meaning that the ADS-B packet contains no error. Therefore, if the received SNR of an ADS-B packet is known, it is possible to know if the timestamp on the packet can be used for PE or not. Based on the simulation result in Figure 2, the time-stamp on ADS-B packet can be used for PE only if it is received at SNR above $20 \mathrm{~dB}$.

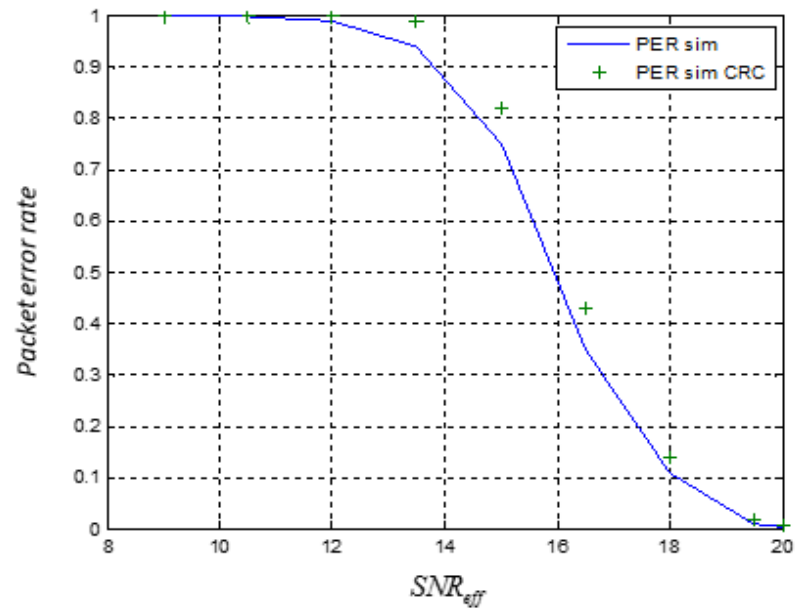

Figure 2: PER versus SNR performance for the ADS-B receiver [15]

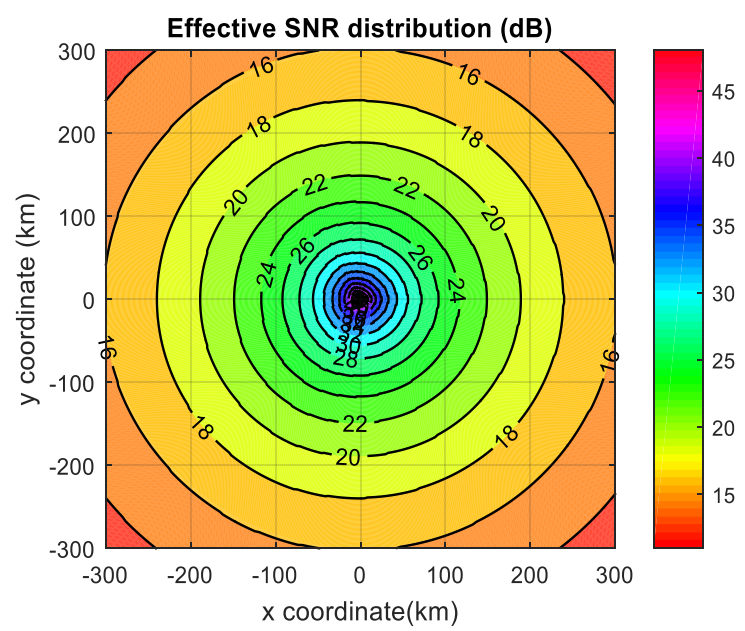

Figure 3: Effective SNR at each emitter position [5 km separation]

\subsection{Effective Snr Versus Emitter Position:}

The ADS-B packets were received at difference ADS-B receivers with slight differences in the SNR value. Thus, the effective SNR was obtained by taking the minimum SNR from all the ADS-B receivers [22]. For each emitter position, the received SNR was calculated based on emitter transmit power of 250 watts, receiver sensitivity of $-90 \mathrm{dBm}$, transmitter and receiver antenna gain of $3 \mathrm{dBi}$ and $12 \mathrm{dBi}$ respectively assuming a free space path loss model. The transmitter/receiver parameter are selected based on actual system used in the aviation industry [23, 24]. Figure 3 shows the effective SNR for emitter positions within a $300 \mathrm{~km}$ coverage from the center of ADS-B receivers in a triangular configuration at altitude of $1 \mathrm{~km}$. From Figure 3, the effective SNR depends on the emitter range at which the ADS-B packet is transmitted from the center of the ADS-B receiver configuration. It decreases with increase in emitter range from $0 \mathrm{~km}$ to $200 \mathrm{~km}$. At constant emitter range, there is no significant difference in the effective SNR between emitter at altitude $1 \mathrm{~km}$ and $15 \mathrm{~km}$. From Figure 2 and Figure 3, it can be concluded that 3-D TOA based PE is possible using the minimum number of ADS-B receiver if only the emitter is located within a coverage range of $190 \mathrm{~km}$

\subsection{Emitter PE Error:}

In section 4.2 , it was established that using the ADS-B receiver in [15], 3-D TOA based PE is possible only for emitter within $190 \mathrm{~km}$ coverage radius from the center of the ADS-B configuration. Error in the TOA measurement results to error in estimating the emitter position. Using a time-stamp resolution of $18 \mathrm{nsec}$ (5.4 meters) [17], the TOA based position error at each emitter position is determined.

Figure 4 shows the PE RMSE of the TOA based system for emitter at altitude of $1 \mathrm{~km}$ using the ADS-B receiver triangular configuration shown in Figure 1. It can be seen that the PE RMSE is position dependent. For both the horizontal and altitude PE, the error increases with increase in emitter range from $0 \mathrm{~km}$ to $200 \mathrm{~km}$. At emitter position $\left(R=50 \mathrm{~km}, \theta=90^{\circ}, z=1\right) \quad$ in cylindrical coordinate system which corresponds to $(x=0 \mathrm{~km}, y=50, z=1) \quad$ in the rectangular coordinate system using Eq. (17), the horizontal and altitude RMSE are $0.025 \mathrm{~km}$ and $0.75 \mathrm{~km}$ respectively. At emitter position $(x=-145 \mathrm{~km}, y=-145, z=1)$, the horizontal and altitude RMSE respectively are 0.11 $\mathrm{km}$ and $2.1 \mathrm{~km}$. Comparison between the horizontal and altitude RMSE shows that the altitude RMSE is about $2 \mathrm{~km}$ higher than the horizontal RMSE. This is 
due to the use of the second order quadratic, Eq. (13), that results to square magnitude error in estimating the altitude of the emitter. The use of additional ADS-B receiver for the $\mathrm{PE}$ process eliminates the square magnitude error as it results in four TOA equations. Further simplification of these equation will result in 3 equations having 3 unknowns which are the emitter positions $(x, y, z)$. Least square (LS) method can then be used in solving for the emitter position thus, eliminating square magnitude error. Based on the ADS$B$ receiver in section 4.1, the maximum horizontal and altitude error expected are $0.12 \mathrm{~km}$ and $2.2 \mathrm{~km}$ respectively within the $190 \mathrm{~km}$ coverage.

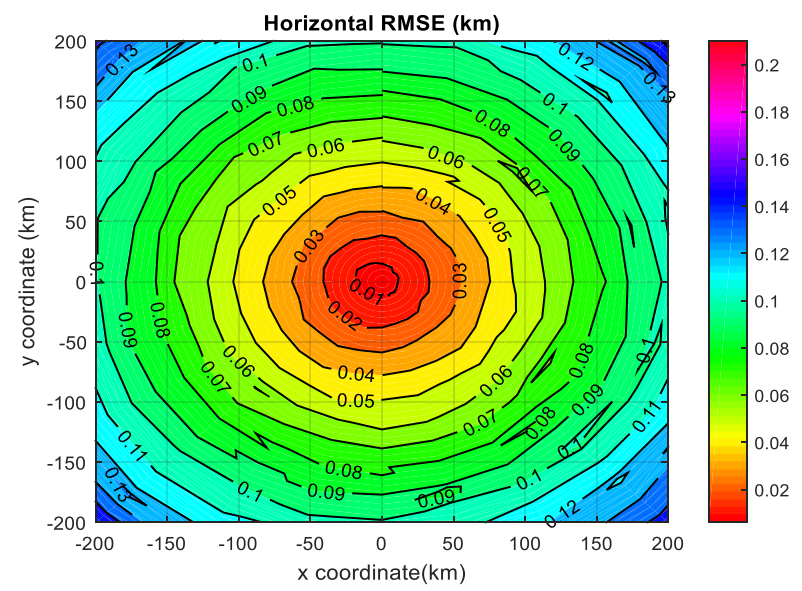

(a) Horizontal RMSE error

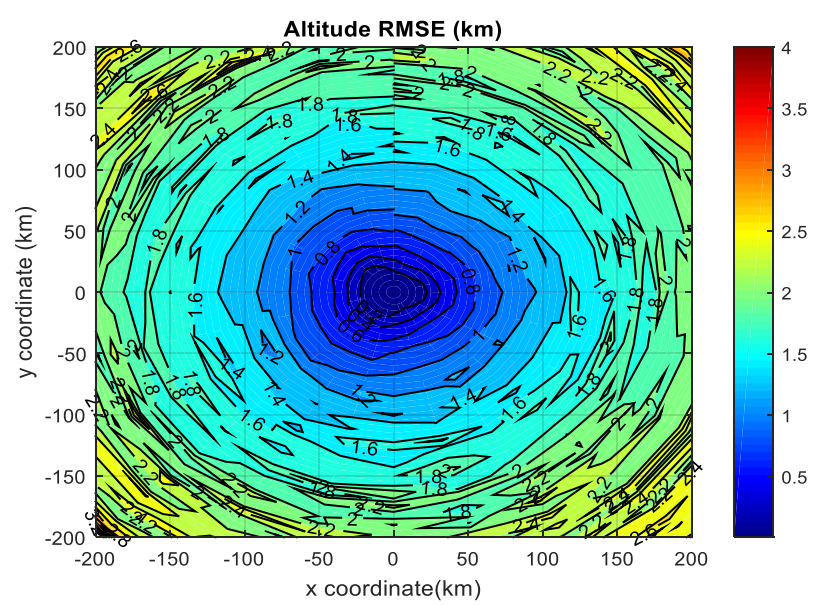

(b) Altitude RMSE

Figure 4: TOA based PE RMSE for emitter at $1 \mathrm{~km}$ altitude with $A S B-B$ in triangular configuration

\section{CONCLUSION}

In this work, a 3-D linear TOA based PE algorithm for an emitter using the minimum number of receiving station was developed. Using an ADS-B receiver at the receiving station, the emitter PE accuracy of the TOA based system was determined with the ADS-B receivers in a triangular configuration. The ADS-B receiver can receive and validate ADS-B packet sent from an emitter within $190 \mathrm{~km}$ coverage radius that is $P E R=0$. The result also shows that the $\mathrm{PE}$ algorithm using the minimum number of ADS-B receivers for the 3-D has altitude estimation error $2 \mathrm{~km}$ higher than the horizontal PE which is due to the use of the second order quadratic equation for the altitude estimation process. Increase in the number of receiving stations will increase the accuracy in both the horizontal and altitude estimation.

\section{REFERENCES}

[1] Duran, M. A. C., D'Amico, A. A., Dardari, D., Rydström, M., Sottile, F., Ström, E. G. and Taponecco, L.,"Terrestrial Network-Based Positioning and Navigation," in Satellite and Terrestrial Radio Positioning Techniques, pp. 75153, 2012.

[2] Galati, G., Leonardi, M., Magaro, P., and Paciucci, V. "Wide area surveillance using SSR mode S multilateration: advantages and limitations," European Radar Conference, 2005. EURAD 2005., Paris, 2005, pp. 225-229.

[3] Galati, G., Leonardi, M., Marco, P. D., Mene, L., Magaro, P., and Gasbarra, M., "New time of arrival estimation method for multilateration target location," International symposium on Sensors and Systems for Airport surveillance (JISSA), 2021, Paris France, pp. 1-11, 2005.

[4] Haneda, K., Takizawa, K. I., Takada, J. I., Dashti, M., and Vainikainen, P., "Performance evaluation of threshold-based UWB ranging methods - Leading edge vs. search back -," 2009 3rd European Conference on Antennas and Propagation, Berlin, , pp. 3673-3677, 2009.

[5] Steffes, C., Kaune, R., Rau, S., and Fkie, F., "Determining Times of Arrival of Transponder Signals in a Sensor Network using GPS Time Synchronization," in Jahrestagung der Gesellschaft für Informatik, Berlin, 2011. [Online]. Available: http://www.user.tu-berlin.de/komm/CD/ paper/100123.pdf. Accessed: Sep. 27, 2016.

[6] Li, Y. C., Go, S., Yoo, H. R., Kim, S. D., and Chong, J. W., TOA Estimation Algorithm Based on ShiftInvariant Technique for Multi-Band Signals. AETA 2013: Recent Advances in Electrical Engineering and Related Sciences, Springer Science + Business Media, pp. 241-246, 2013.

[7] G. Morrison and M. Fattouche, "Super-resolution modeling of the indoor radio propagation channel," IEEE Transactions on Vehicular Technology, vol. 47, Number 2, pp. 649-657, 1998. 
[8] Oh, D., Li, Y.C., Liu, S., Yoon, S.H. and Chong, J.W., "Dual shift invariant TOA estimation algorithm for multi-band signals," IEEE Communications Letters, vol. 16, Number 10, pp. 1568-1571, 2012.

[9] Li, X. and Pahlavan, K., "Super-resolution TOA estimation with diversity for indoor Geolocation," IEEE Transactions on Wireless Communications, vol. 3, Number 1, pp. 224-234, 2004.

[10] Mensing, C. and Plass, S. "Positioning algorithms for cellular networks using TDOA," Proc. IEEE International Conference on Acoustics, Speech and Signal Processing, Toulouse, France, May., pp. 513 - 516, 2006.

[11] Fenwick, A. J. "Algorithms for position fixing using pulse arrival times," IEE Proceedings - Radar, Sonar and Navigation, vol. 146, Number 4, 1999, p. $208-212$.

[12] Yaro, A. S., Musa, M. J., Sani, S. and Abdulaziz, A., "3D Position Estimation Performance Evaluation of a Hybrid Two Reference TOA/TDOA Multilateration System Using Minimum Configuration". International Journal of Traffic and Transportation Engineering, vo.l 5, Number 4, pp.96-102, 2016.

[13] Sha'ameri, A. Z., Shehu, Y. A., and Asuti, W. "Performance analysis of a minimum configuration multilateration system for airborne emitter position estimation," Defence $S$ and $T$ Technical Bulletin, vol. 8, Number 1, pp. 27-41, 2015.

[14] Yaro, A. S. and Zuri, S. A., "Mathematical Model of Position Estimation Error for a Multiangulation System," in the 6th International Graduate Conference on Engineering, Science \& Humanity: Empowering innovation and Entrepreneurship for sustainable Development, Universiti Teknologi Malaysia (UTM), Johor Bahru, Malaysia, Universiti Teknologi Malaysia (UTM), pp. 134-136.

[15] Abdulaziz, A., Yaro, A. S., Adam, A. A., Kabir, M.T. and Salau, H. B., "Optimum receiver for decoding automatic dependent surveillance broadcast (ADS-B) signals," American Journal of Signal Processing, vol. 5, Number 2, pp.23-31, 2015.
[16] Neven, W. H. L., Quilter, T. J., Weedon, R. and Hogendoorn, R. A., "Wide area multilateration," NLR, Report on EATMP TRS 131, 04, 2005.

[17] Agilent Technologies, "N6841A RF Sensor for Signal Monitoring Networks." online: http://cp.literature.agilent.com/litweb/pdf/5990 -3839EN.pdf, March, 1st, 2017.

[18] Monteiro, M., Barreto, A., Kacem, T., Wijesekera, D. and Costa, P., "Detecting malicious ADS-B transmitters using a low-bandwidth sensor network," 18th International Conference on Information Fusion (Fusion), Washington, DC, July 2015, pp. 1696-1701, 2015.

[19] Strohmeier, M., Schäfer, M., Lenders, V. and Martinovic, I., "Realities and challenges of nextgen air traffic management: the case of ADS-B," IEEE Communications Magazine, vol. 52, Number 5, pp. 111-118, 2014.

[20] Geyer, M. "Aircraft Navigation and Surveillance Analysis for a Spherical Earth," Federal Aviation Administration Wake Turbulence Research Office, Oct. 2014. [Online]. Available: http://ntl.bts.gov/lib/53000/53100/53123/DOT -VNTSC-FAA-15-01.pdf. Accessed: Mar. 2017.

[21] Chen, Y., Francisco, J., Trappe, W., and Martin, R., "A Practical Approach to Landmark Deployment for Indoor Localization," in 3rd Annual IEEE Communications Society on Sensor and Ad Hoc Communications and Networks, 2006, pp. 365373, 2006.

[22] Stein, S., "Algorithms for ambiguity function processing," IEEE Transactions on Acoustics, Speech, and Signal Processing, vol. 29, Number 3, pp. 588-599, 1981.

[23] SELEX Sistemi Integrati: ADS-B Subsystem, Standard E5010015201SDD, 2014.

[24] SAAB, "Multilateration System High Performance Positioning on the Ground and in the Air," [Online]. Available: http://saab.com/security/airtraffic-management/air-trafficmanagement/Wide-Area-Multilateration/. Accessed: Nov. 20, 2016. 